\title{
Ammonium nitrate induced cracking of a dryer.
}

\author{
R.E.Clegg ${ }^{1 \S}$, R.Tomlins ${ }^{2}$ and M.J.Mackay ${ }^{3}$ \\ 1. Process Engineering and Light Metals Centre, Central Queensland University, P.O.Box \\ 1319, Gladstone, QLD 4680 Australia. \\ 2. Rexsan, P.O.Box 1024, Gladstone QLD 4680, Australia. \\ 3. Orica Mining and Resources, Yarwun Industrial Estate, Gladstone, QLD 4680
}

Australia.

§ corresponding author, r.clegg@cqu.edu.au

\begin{abstract}
After less than one year of service, extensive cracking of two ammonium nitrate prill dryers was found. The cracking was found to be ammonium nitrate-induced stress corrosion cracking and the failure was attributed to a lack of post-weld stress relieving of the fabricated structures. It was considered likely that the SCC would occur more slowly once the cracks had progressed beyond the region of high residual stresses and that the design operating stresses in the dryer would be insufficient to cause rapid cracking in the dryers. It order to manage the dryers until new ones could be fabricated, the dryers were operated normally with regular inspections to monitor crack growth and to date, no significant addition crack growth of major cracks was observed.
\end{abstract}

\section{Introduction}

Ammonium nitrate (AN) is used as a fertilizer and as an oxidant for explosives. There is currently a high demand for AN, particular for use as an explosive in coal mines and plants in Australia have been running at capacity. As part of the process of producing AN, a prill, or small pellet of AN, is formed and then dried in a rotary dryer. This case study describes the cracking of a dryer in an AN plant by stress corrosion cracking. This dryer was one of two dryers that had been installed to dry AN from a moisture content of 5\% to the final product value. It was found that both dryers were cracked, although one more severely than the other. This study represents the analysis of the more severely cracked dryer. The other dryer showed similar features, although to a lesser extent as it was younger. Failure of a key piece of 
equipment such as an AN dryer can lead to significant financial loss, particularly from the downtime associated with the failure. In this instance the dryer had been operating for only one year at the time the cracking was observed. The dryers had replaced an earlier dryer, but some significant design changes had been carried out, particularly with respect to the mounting of the bearings.

The rotary dryer consisted of a long horizontal tube that rotated about its axis and was mounted on two external bearings. The tube was approximately $3 \mathrm{~m}$ in diameter and $\mathrm{AN}$ was fed from one end and was removed from the other. The tube had been fabricated from boiler plate and had a number of longitudinal and transverse welds in the structure. The dryer received moist $\mathrm{AN}$ at one end and this was dried by a blast of warm air. The temperature of the AN as it entered the dryer was approximately $50^{\circ} \mathrm{C}$ and the dry air was at about $70^{\circ} \mathrm{C}$. In the design of dryer used in this case, the dryer air came from both ends of dryer and was vented in the middle. On the inside of the dryer were a number of lifting lugs which were used to roll the prill in the drying air. The problem was first encountered when it was noticed that a number of the lifters had broken away from the shell of the dryer. The dryer was then inspected and it was found that a significant number of lugs were cracked and that the cracking extended into the shell.

\section{Examination of the dryer}

An extensive magnetic particle inspection (MPI) program was instigated to determine the internal state of the dryer. The dryer was a rotating cylindrical dryer that contained internal lifters. In the region where most of the cracking had occurred there was no coating on the internal surface of the dryer, even though there was some coating at the end of the dryer where 
the prill was fed. The dryer had two main sections separated by a central flue. A large number of cracks were found on the internal surface particularly towards the flue on the inlet side of the dryer, although cracking was found throughout the dryer where bare metal was exposed to prill.

Cracking of the dryer shell was generally associated with welds or in several cases, where welds had been removed. Cracking of the lugs was generally either in the toe of the welds or transverse to the welds (see Figure 1). Cracking was also found associated with the circumferential and longitudinal welds in the shell (see Figure 3). In some cases, the cracks penetrated the thickness of the shell. The cracks were predominantly transverse to the welds and often were confined to the weld metal. However, in a number of cases, the cracks were found predominantly in the parent plate.

One region where the longitudinal weld was cracked was removed from the shell for examination. This was considered to be representative of the type of cracking found in the shell.

Careful examination of the inner surface using MPI suggested that there was extensive microcracking of the inner surface of the inside of the dryer, even where welds were not present. This microcracking was only faintly visible using the MPI but was confirmed by later metallographic examination and was found to be intergranular corrosion.

\section{Examination of the Lugs}

Each of the internal lifters was attached to the shell by bolting to two welded lugs. In a number of cases, these lugs had fractured through the heat affected zones of the welds. Failure of the lugs had occurred in the region of the welds, predominantly in the HAZ (see Figure 2). The 
fracture showed little ductility, although it was covered with a layer of corrosion product. The fracture did not appear to be a fatigue failure and the fracture surface was textured in a manner consistent with stress corrosion cracking.

The failed section of welded lugs was examined metallographically in two places. Specimens were mounted in polymethylmethacrylate (PMMA) and prepared to ASTM E3, prior to examination at magnifications up to x1000 in the un-etched condition and after etching in 2\% nital. Both regions showed a number of branched, intergranular cracks typical of stress corrosion cracking. The steel microstructure consisted of ferrite and pearlite in a manner typical of low carbon structural steel.

\section{Examination of circular test section}

A $200 \mathrm{~mm}$ diameter circular test section containing a longitudinal weld was cut from the shell of the dryer. Several large transverse cracks (approximately $50 \mathrm{~mm}$ ) were found in the weld metal and these cracks extended into the parent metal. In addition, a number of cracks that were smaller in size but entirely confined to the parent metal were also found. Metallographic sections of a weld metal crack and one of the smaller non-weld metal cracks were taken.

The weld metal consisted of fine pearlite and ferrite. Cracking of the weld metal was branched and intergranular and occurred from the inside surface of the dryer. The surface layers of the weld metal on the inside of the dryer showed extensive intergranular corrosion.

The parent plate consisted of pearlite and ferrite. In the section examined, the crack had penetrated from the inside surface of the shell to the outside surface. In places the crack had branched and ran parallel to the plane of the plate. This was particularly noticeable near the centreline of the plate. Cracking was generally intergranular in nature and were commonly 
filled with oxide. A number of fine intergranular cracks could be seen propagating from the inside surface of the shell (Figure 4). The inside surface also showed evidence of general corrosion and some intergranular attack (Figure 5).

The results of the Vickers hardness testing of the parent plate are as follows:

$$
140 \quad 138 \quad 135 \quad 140 \quad 137 \quad \text { Ave }=138 \text { HV10 }
$$

\section{Immersion Tests}

The inside surface of the dryer showed evidence of intergranular attack. In order to confirm that this was due to AN attack, samples of four alloys were immersed in a solution of concentrated $\mathrm{AN}$ at $80^{\circ} \mathrm{C}$ for 8 weeks. The materials were structural carbon steel boiler plate, bisalloy 80 (a quenched and tempered steel plate), 316 stainless steel and 17-4 PH stainless steel. This test was used as a screening test to evaluate the relative susceptibility to attack. The immersion test was carried out without potential control and the samples were electrically isolated from one another, apart from the solution.

After the immersion test, the samples were mounted in PMMA and were examined metallographically. The two stainless steel samples (316 and 17-4 PH) showed no evidence of corrosion, either visually or in the metallographic sections. The boiler plate had corroded significantly and showed intergranular attack on the metallographic sections. The intergranular attack was similar to that found in the dryer. The bisalloy 80 plate also evidence of corrosion and on the metallographic section, showed preferential corrosion along martensite laths. These tests confirmed that in unloaded steel boiler plate, intergranular corrosion was possible in boiler plate as a result of attack from concentrated AN. 


\section{Discussion}

\subsection{Failure root cause}

Failure of the dryer was by stress corrosion cracking (SCC). This was identified by the metallurgical examination, which found branched intergranular cracking which is typical of SCC. The material microstructure and hardness were typical of low-carbon structural steel and no metallurgical defects likely to significantly contribute to the failure were found. Stress corrosion cracking is caused by the combined effects of stress and a corrosive environment. In order for SCC to occur, the material of construction must be susceptible to SCC in that environment and susceptibility may be quite specific.

Ammonium nitrate has been observed to cause SCC in a variety of steels, including mild steel, in other applications[1-9]. It is possible for ammonium nitrate to accumulate in waste heat boilers in power stations. At low temperatures where free moisture is present, it is know that high levels of ammonium nitrate can become dissolved in the moisture and can readily produce SCC in carbon and low alloy steels [10]. The ASM Handbook reports susceptibility at $60^{\circ} \mathrm{C}$. The situation found in the dryer is similar to the cases where SCC is found in power stations, in that the temperatures are low and the ammonium nitrate, whilst solid is still moist. In some ways it is more severe because of the amount of ammonium nitrate present.

Information in the open literature on ammonium nitrate induced stress corrosion cracking in mild steels is limited. Krautschick et al. [11] examined the effect of phosphorous on SCC of mild steels in nitrate solutions and found that although increased P content increased the susceptibility to SCC, mild steels with low P would still crack. Cahoon [9] characterised corrosion behaviour of mild steel in urea-ammonium nitrate solutions and found that as temperature was increased above $60^{\circ} \mathrm{C}$ the corrosion rate increased with the steel exhibiting 
active-passive behaviour. Stress corrosion cracking is sometimes associated with activepassive behaviour[1] and hence this may signal that SCC is more likely at temperatures above $60^{\circ} \mathrm{C}$.

The shell near the entrance of the AN had been coated, but the coating did not extend all the way to the flue. Therefore, there was bare metal in the region where the cracking occurred. Throughout the dryer where bare metal was present, some level of cracking had occurred. The prill had a low moisture content as it entered the dryer (approximately 5\%) and the drying air also had been chilled prior to heating in order to ensure that it was as dry as possible. Nonetheless there must have been some build-up of moisture on the shell surface, which would have become saturated with AN. It is not possible to completely exclude moisture from the dryer, as periodically it is cleaned and washed with water. However, it may be that condensation of moisture during the drying process is the driver of the cracking rather than wash water.

The pattern of cracking observed suggested that the stresses that drove the SCC cracks resulted from residual stresses produced by welding. Because of a concern that stress relieving would have caused the shell to warp, which could not be tolerated by the new design of the bearings, the structure had not been stress relieved after fabrication. If welds that have not been stress relieved are put into an environment that can induce SCC, transverse cracking of butt welds may occur and this is observed here. In fillet welds, the residual stresses may also be high in the toe of the weld and much of the cracking of the shell associated with the lug welds was in the toe region. It is interesting to note that even when welds had been removed cracking was still severe as the residual stresses associated with the welding had not been removed. 
Whilst most of the cracking observed here can be explained in terms of the residual stresses caused by the welds, some the cracking was a little more unusual. In particular, some cracking was found in a direction that was transverse to the butt welds but in the parent plate centred approximately $25 \mathrm{~mm}$ from the fusion line. One possible reason is that the plates had been formed at low temperature and the springback of the rolled plate once formed into the shell caused tensile residual stresses on the inside of the shell. These appear to have been been sufficient to drive SCC cracks. The extra stresses imposed on the plate by the welding may have increased the likelihood of cracking adjacent to the weld, but it is possible that the residual stresses in the rolled plate may be sufficient on their own to initiate cracking in the longer term.

From the metallurgical examination, it was apparent that there was considerable intergranular attack on the inside surface of the shell. The immersion tests indicated that the intergranular corrosion occurred even in the absence of applied stress. There then appeared to be two mechanisms operating. The entire exposed inner surface of the dryer had probably developed a level of intergranular corrosion. However, in general the intergranular corrosion became self-limiting, in that the intergranular defect became filled with corrosion product and this tended to slow the intergranular penetration as it became deeper. However in cases where high residual stresses were present, this intergranular penetration would provide an initiation point for the stress corrosion cracking and probably was the precursor for the SCC. It is postulated that the SCC was not initiation limited in this case, but the intergranular corrosion provided ready nuclei for the SCC. 


\subsection{Failure management}

There are two main methods of reducing SCC in this type of application. First, the sources of high stresses should be eliminated. It does not appear that in this case, the SCC was driven by the design stresses in the shell. The main stresses driving the SCC were residual stresses from both the welding and the forming of the shell and if these stresses were eliminated, then the main driver for SCC would have been removed. Several authors have explored methods for the elimination of residual stresses during repairs[12, 13], but these methods were not considered to be economically viable in this case. It is therefore important that new dryers be adequately stress relieved prior to going into service. Second, the shell could be coated on the inside in order to prevent contact between the corrosive environment and the steel. If an alternative material is used for the application, careful consideration to its susceptibility to SCC is needed.

Because of the lead time involved in obtaining new dryers, it was necessary to keep the cracked dryers in service, at least until replacements could be sourced. The question arises as to how critical the cracking of the dryer was considering that the largest cracks were of the order of $100 \mathrm{~mm}$ in length. The dryers were not highly stresses and were not internally pressurised and so the design operating stresses in the shell were not likely to be high in the region of cracking. Furthermore, it is possible that the cracking that had occurred had relieved much of the residual stress and the further growth of cracks would be much slower. In numerical modelling studies, a number of authors have shown that the high residual stresses associated with welds can be highly localised[14, 15] and it is likely that once outside of these highly stressed regions, the SCC cracks will stop growing due to the decreased stress levels. 
Thorough cleaning of the inside of the dryer may remove residual AN from the cracks. If this can be done and the inside of the dryer were coated, the further growth of the cracks would be by conventional fatigue. It is envisaged that the low design operating stresses would mean that fatigue crack growth would be very slow. However it is difficult to guarantee that all of the AN will be removed from the cracks and some crack growth may continue by SCC.

Furthermore, if the inside of the dryer were coated, it would be much more difficult to inspect the dryer and monitor the crack growth.

Repair of the cracks was not considered to be feasible, due to the extent of cracking and the further problems that repair welds would introduce. The decision was taken to manage the cracks until a replacement could be made. The failure was managed by continuing to operate the dryers normally during the period taken supply new dryers. A program of periodic inspection was instigated where some of the larger cracks were marked and monitored for crack growth. In the six month period after the cracks were first detected, none of the cracks being monitored had grown significantly, supporting the postulation that the SCC cracks had effectively relieved the welding stresses and that the operating stresses were not significant drivers of the SCC. The only area which showed some evidence of adverse crack growth was the region where the circular sample had been taken. In order to repair the dryer, a new segment of material was welded back into the dryer to replace the material taken. This type of circular patch tends to induce very high residual stresses and was not stress relieved. As a result, significant cracking of the weld was occurring, although this did not affect the operation of the plant. 


\section{Conclusions}

Failure of the dryer was by ammonium nitrate induced stress corrosion cracking, which was occurring at temperatures lower than $70^{\circ} \mathrm{C}$. The main cause of the failure was the failure to stress relieve the dryer after welding. Extensive intergranular corrosion of the inside of the dryer was also observed, but it was concluded that this was essentially benign unless combined with the residual stresses in the welded structure.

\section{Acknowledgements}

The authors wish to thank Orica Australia for permission to publish this case study.

\section{References}

[1] J.A. Beavers, N.G. Thompson, R.N. Parkins. Stress-corrosion cracking of low strength carbon steels in candidate high level waste repository environments: Environmental effects. Nuclear and Chemical Waste Management. 5 (1985) 279-96.

[2] O.R. Brown, G.F. May. The anodic behaviour of iron in acidic solutions of liquid ammonia. Electrochimica Acta. 22 (1977) 1347-56.

[3] G.L. Edgemon, M.J. Danielson, G.E.C. Bell. Detection of stress corrosion cracking and general corrosion of mild steel in simulated defense nuclear waste solutions using electrochemical noise analysis. Journal of Nuclear Materials. 245 (1997) 201-9.

[4] J. Flis. Role of oxide films in stress corrosion cracking of mild steel in nitrate solutions. Corrosion Science. 15 (1975) 553-64.

[5] J.D. Gilchrist, R. Narayan. Environmental cracking of eutectoid steel. Corrosion Science. 11 (1971) 281-96.

[6] T.P. Hoar, J.R. Galvele. Anodic behaviour of mild steel during yielding in nitrate solutions. Corrosion Science. 10 (1970) 211-24.

[7] V. Linton, N. Laycock, D. Keen, P. Boon. SCG failure of a super duplex separator vessel in an ammonium nitrate plant. Materials Performance. 47 (2008) 64-8.

[8] J. Mieluch, M. Smialowski. The behaviour of grain boundaries in iron during anodic polarization in ammonium nitrate solution. Corrosion Science. 4 (1964) 237-43.

[9] J.R. Cahoon. Corrosion characteristics of mild steel in urea ammonium nitrate fertilizer solutions. Corrosion. 58 (2002) 166-74.

[10] W. Cox, Components susceptible to dew-point corrosion, ASM Handbook v.13c Corrosion: Environments and Industries, ASM International, 2006, pp. 491-6.

[11] H.J. Krautschick, H.J. Grabke, W. Diekmann. The effect of phosphorus on the mechanism of intergranular stress corrosion cracking of mild steels in nitrate solutions. Corrosion Science. 28 (1988) 251-8.

[12] A. Aloraier, A. Al-Mazrouee, J.W.H. Price, T. Shehata. Weld repair practices without post weld heat treatment for ferritic alloys and their consequences on residual stresses: A review. International Journal of Pressure Vessels and Piping. 87 (2010) 127-33.

[13] F. Tölle, A. Gumenyuk, M.O. Gebhardt, M. Rethmeier. Post-Weld Residual Stress Mitigation by Scanning of a Defocused Laser Beam. Physics Procedia. 12 (2011) 410-8. 
[14] Z. Barsoum, I. Barsoum. Residual stress effects on fatigue life of welded structures using LEFM. Engineering Failure Analysis. 16 (2009) 449-67.

[15] Z. Barsoum, A. Lundbäck. Simplified FE welding simulation of fillet welds - 3D effects on the formation residual stresses. Engineering Failure Analysis. 16 (2009) 2281-9. 


\section{List of Captions}

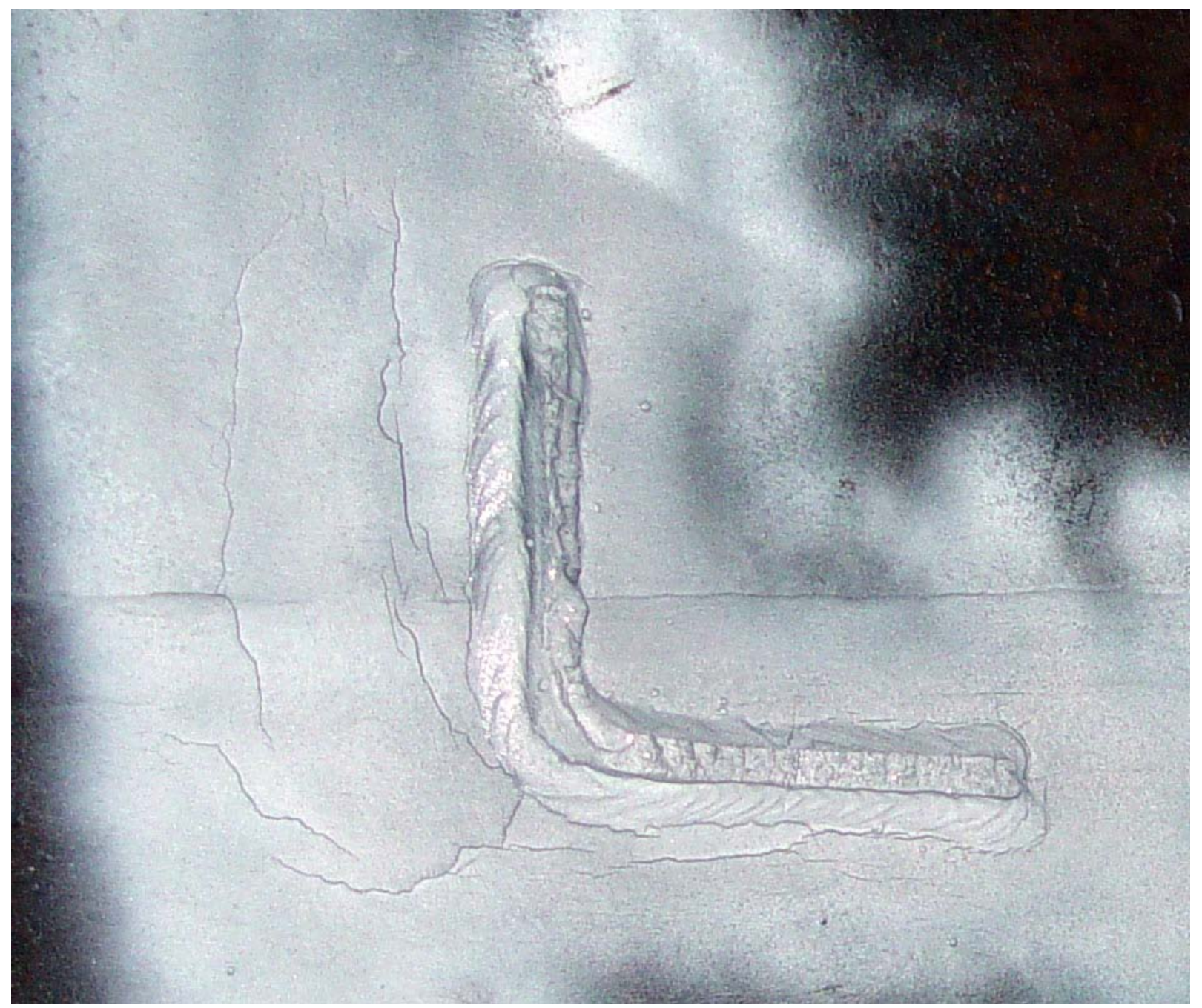

Figure 1 Extensive cracking around the welded lugs and in the welds could be seen on the inside of the shell. 


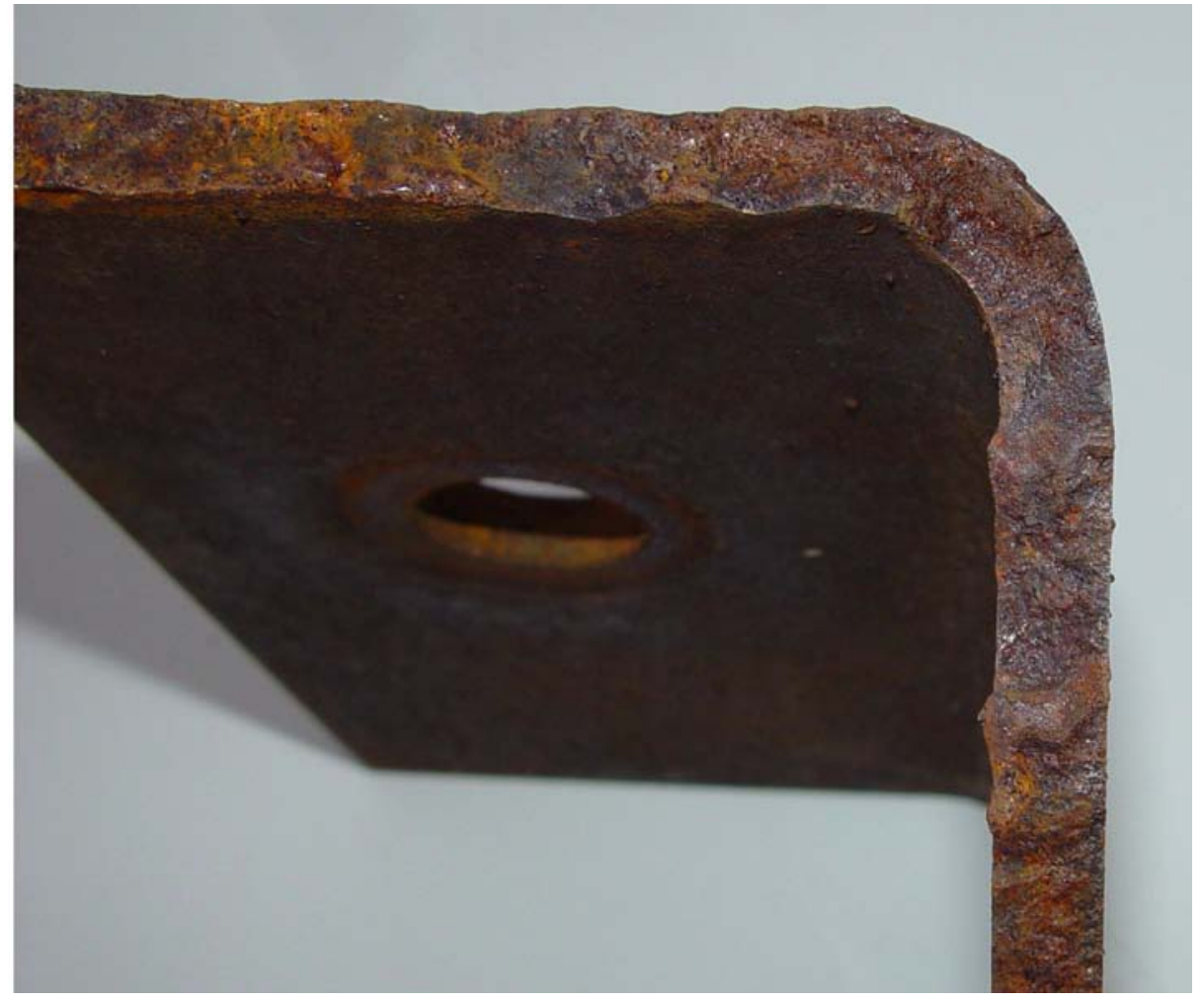

Figure 2 Lifter showing the cracked lugs. These lugs had been welded to the shell and had fractured through the HAZ of the weld. 


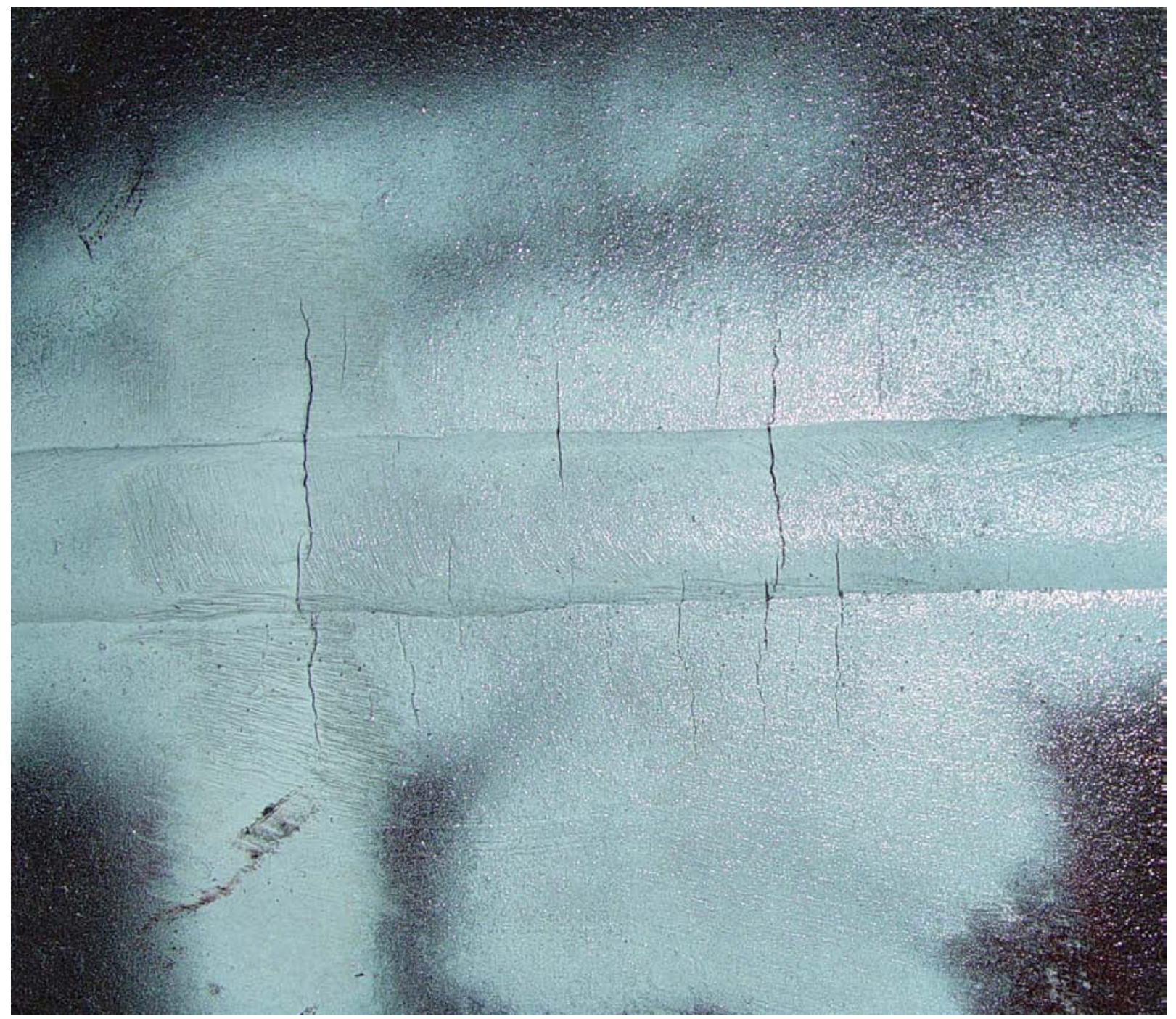

Figure 3 Transverse cracking in seam welds. 


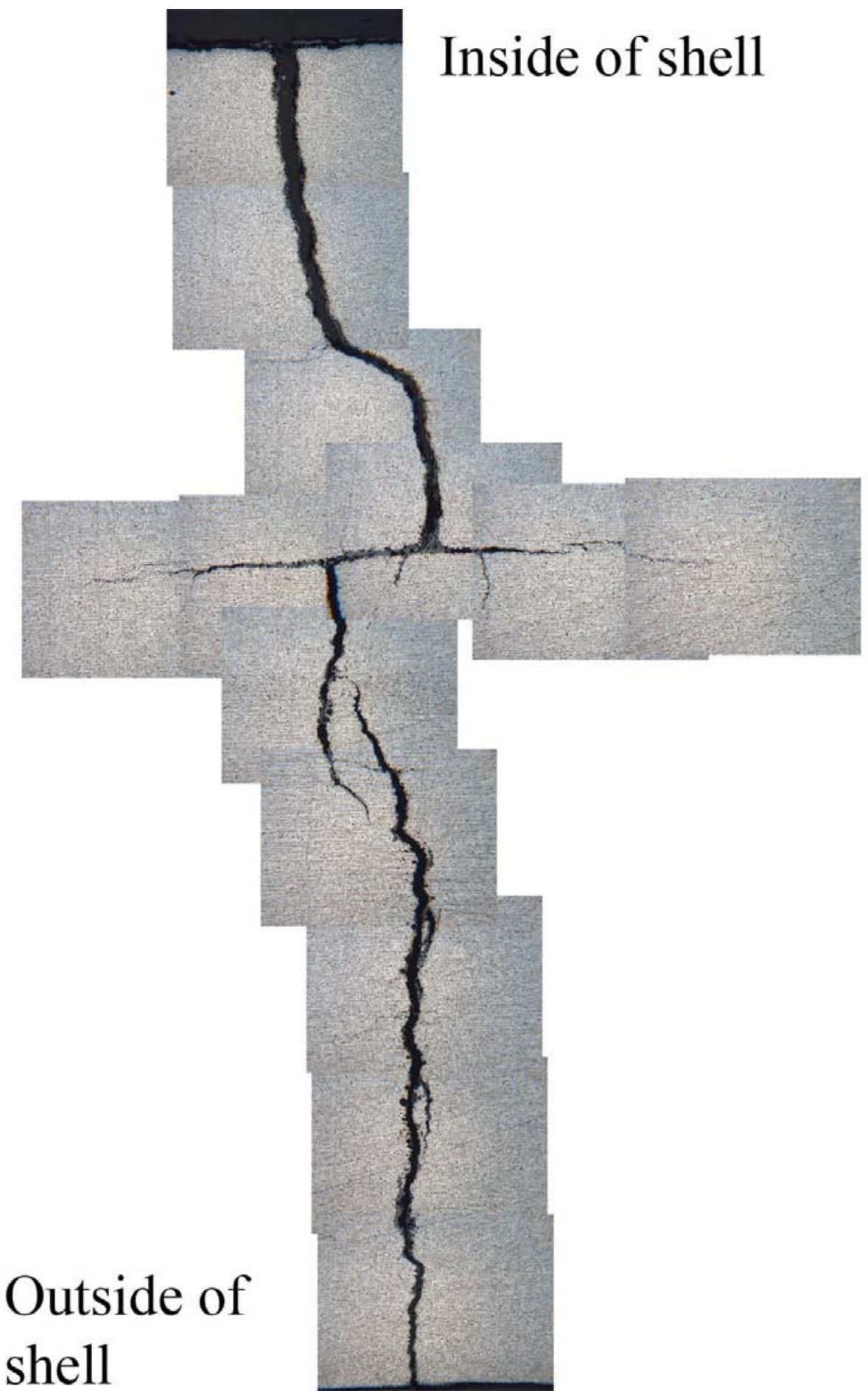

Figure 4 Cracking of the shell occurred from the inside to the outside of the shell. Significant centreline cracking was observed. 


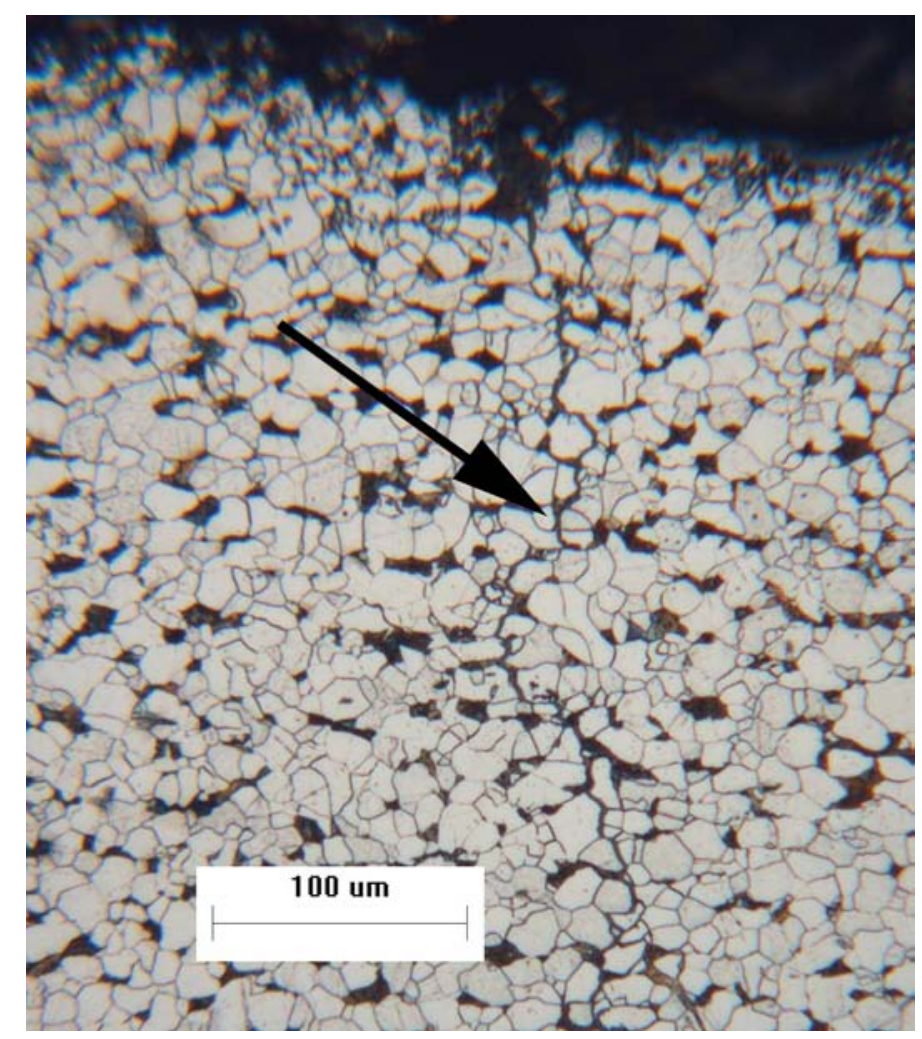

Figure 5 Small crack coming from the surface of the steel plate. 